\title{
Aspects de l'énergie nucléaire dans la perspective d'un développement durable
}

Le rendez-vous d'ENC, organisé tous les quatre ans sous l'égide de l'" European Nuclear Society", est une occasion privilégiée de "prendre le pouls » du nucléaire

mondial. Disons-le sans circonlocutions : la situation actuelle et le développement futur de l'atome civil semblent durablement entrés en zone de turbulences, tout au moins si l'on considère la question à travers le prisme

politique. C'est ce que suggère $P$. Upson, président de l'ENS, en soulignant que le principal danger qui menace aujourd'hui le nucléaire est celui de devenir " un gage

monnayable dans le jeu politique ». Mais au-delà des enjeux de pouvoir et des considérations électorales - et du rôle central conféré, dans ce contexte, aux opinions publiques réelles ou supposées... - le nucléaire doit être aussi appréhendé dans ses réalités objectives, techniques, économiques, environnementales. Etabli à partir des communications présentées lors d'ENC'98 (qui s'est tenu fin octobre à Nice) le dossier présenté dans ce numéro aborde l'ensemble de ces paramètres et situe le nucléaire par rapport aux autres sources d'énergie dans

la perspective du développement durable. Par quelque

biais que l'on mène la comparaison, le nucléaire y apparaît à son avantage, surtout si l'on considère la nécessité de satisfaire, dans le futur, à une exigence aux termes contradictoires : produire des quantités massives

d'électricité supplémentaires en respectant les limites d'émission de gaz à effet de serre fixées par les accords de Kyoto. Au-delà des controverses du moment, la poursuite de cet objectif par la communauté mondiale donne au nucléaire un rôle de premier plan sur la scène énergétique de demain.

F. Sorin 